Original scientific paper

\title{
NUMERICAL SIMULATION OF MIGRATION LAWS OF DENSE PARTICLE FLOW IN PIPELINES
}

\author{
Fu, S. ; Guo, X. Y.*; Dong, L. H. ${ }^{* * \# ; ~}$ Sheng, K. ${ }^{* * *} \&$ Sun, A. ${ }^{* * * *}$ \\ * School of Civil Aviation, Zhengzhou University of Aeronautics, Zhengzhou, 450046, China \\ ** College of Safety Engineering, Shenyang Aerospace University, Shenyang, 110136, China \\ ${ }^{* * *}$ Shenzhen Urban Public Safety and Technology Institute, Shenzhen, 518038, China \\ * School of Aerospace, Transport and Manufacturing (SATM), Cranfield University, Bedfordshire, \\ MK43 0AL, UK \\ E-Mail: 20180018@ sau.edu.cn $\left({ }^{\#}\right.$ Corresponding author)
}

\begin{abstract}
To explore the migration distribution laws of the cleaning agent of solid powder in industrial ventilation pipelines, a gas-solid flow migration model based on dense discrete phase model (DDPM) was built. Influences of various material injection methods (injection and jetting methods), positions, and directions in the narrowing zone of pipelines based on the migration laws of particulate cleaning agents in the pipelines were analysed. Results demonstrate that under the top injection method in the narrowing zone, the particulate cleaning agent presents obvious flow characteristics along the pipeline walls and cleaning agent concentration distributes unevenly in the pipelines. After the injection method is changed to top jetting, particle concentration remains high at one end near the wall and low at the other end. After changing the mouth from the top of the narrowing zone to the middle section and changing the spraying direction from perpendicular to the mainstream direction to the horizontal, the particle concentration of the cleaning agent reaches uniform distribution near the mouth and at the pipeline ends. Particle concentration in the pipelines is improved significantly.

(Received in November 2021, accepted in February 2022. This paper was with the authors 1 week for 1 revision.)
\end{abstract}

Key Words: Dense Discrete Phase Model, Pipelines, Dense Particle Flow, Numerical Simulation

\section{INTRODUCTION}

Waste gas is a by-product of industrial production. In general, waste gases often contain many toxic and harmful substances and may cause heavy pollutions and an environmental impact if discharged into air directly [1]. Thus, some processing technologies are needed to purify industrial waste gas effectively before discharge. Choosing a scientific and reasonable waste gas processing technology not only can purify more industrial pollution substances in a shorter period but also can further lower the economic cost of industrial waste gas processing, thereby assuring quality and effect of waste gas disposal [2,3].

At present, sulphur-containing waste gas is generally removed through wet desulfurization and dry desulphurization methods [4-10]. Specifically, the dry desulfurization method uses powder or particulate adsorbent and removes sulphur-containing waste gas through physical adsorption or chemical absorption of solid adsorbent. This is a characteristic of free waste acid emission and small secondary pollution. The wet desulfurization method has been widely applied in all countries. This method washes the waste gas that contains $\mathrm{SO}_{2}$ with the solution. It is characterized by high desulfurization efficiency and the use of simple equipment, and the obtained by-products allow secondary utilization. This method can oxidize $\mathrm{SO}_{2}$ in smoke into $\mathrm{SO}_{3}$, and then condense it into sulfuric acid or reduce $\mathrm{SO}_{2}$ into sulphur, which is further condensed for reuse. Nitric oxides in industrial waste are often processed by catalytic reduction, solution absorption, and adsorption methods [11-15]. Although the catalytic reduction method aims to reduce nitric oxide into $\mathrm{N}_{2}$ under the effect of catalyst, which has high removal efficiency and stable operation, the investment cost is high. The solution absorption method 
aims to absorb nitric oxides in waste gas with compounds such as nitrate, but the absorption efficiency is not high and has poor efficiency in processing waste gas with high nitric oxide content. Adsorption method achieves a high removal efficiency of nitric oxides, but it incurs a high consumption of adsorbent and high consumption of power for equipment operation. Thus, solid adsorbent has extremely wide applications in industrial waste gas processing as a universal waste gas processing technique with low-cost, high efficiency, and small pollution. Various solid adsorbents can be prepared according to different properties of pollutants during use, thereby improving gas adsorption efficiency.

For processing of pollutants in large ventilation pipelines of enterprises, airflow rate and speed in pipelines are high. In this case, solid adsorbent powder is usually injected into pipelines. Solid particulates scatter into the entire pipeline by taking advantage of airflow structures in pipelines, and the retention time of solid cleaning agents in pipelines is prolonged, thereby improving the absorption efficiency of solid particulate cleaning agent without influencing normal airflow. In practical engineering, both injection ways and positions of solid cleaning agent powder can significantly influence the distribution of solid cleaning agent in pipelines, thereby further influencing the dosage of solid cleaning agent and pollutant disposal capacity. On this basis, influences of injection ways and directions of solid cleaning agent on distribution laws and purifying effect of solid cleaning agent were investigated from the perspective of distribution law of solid powder in pipelines. Research conclusions provide references for industrial waste gas processing in practical engineering.

\section{STATE OF THE ART}

The fluid problems in industrial production often use numerical simulation methods to carry out theoretical research [16]. The migration and propagation process of particulate cleaning agent powder along pipelines under airflows belongs to gas-solid flow process. From this perspective, many theoretical, experimental, and numerical simulation studies have been reported. Ahmadi and Goldschmidt [17] conducted various approximations to the Basset equation of particle motion in viscous fluid to solve the complicated dispersion in turbulent fluid. Hutchinson et al. [18] proposed a particulate and drop sedimentation model in turbulent airflows, which reflects the possibility that eddy and particle movement are not completely the same. Moreover, Hutchinson et al. [18] concluded from an experiment that deposition rate of particles was determined by Reynolds number, particle-to-pipe diameter ratio, gas-to-particle density ratio, and distance to the bottom of pipelines. Tian and Ahmadi [19] conducted a series of numerical simulations on transportation and sedimentation of nanometre and micrometre particles in turbulent pipelines by using different turbulence models and the fluent user-defined program, and analysed the trajectory of particles. Tsuji et al. [20] calculated the trajectory of a single particle through the motion equation and conducted numerical simulation of solid pneumatic transmission process in horizontal pipelines. In this study, hydraulic resistance and lift force generated by rotation of particles as well as torque on rotating particles were considered, and the frictional loss generated by the effects of particles and pipe wall was calculated by the impulsive equation. Wu et al. [21] implemented a numerical study on 3D gassolid flowing characteristics of a new spouted-bed structure under the effect of longitudinal eddy by combining the two-fluid model and theory of particulate hydromechanics. Results demonstrate that vertical eddy can strengthen radial mixing of particles and gas phase in the space between spouted area and annulus in the centre of the bed. Zhou et al. [22] established gas-solid flow control equation based on the Euler dual-fluid model and simulated the pressure distribution and dust dispersion process of air-flow field in the fully mechanized coal mining face under different air outputs, thereby providing references to the field dust removal. Li and Kuipers [23] calculated and analysed influences of pressure on the flow structure of dense gas 
fluidized bed and flow pattern changes by using the discrete particle simulation (DPS) method, and analysed particle-fluid interaction through the particle level simulation to highlight the internal mechanism of gas-solid flowing mode. Zhou et al. [24] conducted numerical simulation on particle discrete mechanism in gas-solid jet of burners with rich or poor fuels based on Fluent software by combining discrete element method and computational fluid mechanics, and processed the particle-particle and particle-wall interactions by discrete element method. Meanwhile, influences of separation performances under rich or poor fuels were evaluated. Liu [25] conducted a numerical simulation on sedimentation and diffusion of fine particles on the wall surface, thereby disclosing their propagation laws on the wall surfaces of straight square pipes and bending pipes. These results provide insights into the propagation laws of fine particles on pipelines. Zhang [26] conducted a numerical simulation on the propagation laws of particles in kitchens where several other factors were considered. The indoor propagation of particles was simulated by using the discrete particle model in the Lagrange method. According to analysis, motions and distributions of three types of particles with different sizes in the kitchen were discovered.

Although the preceding studies have involved migration and propagation of particle flows in ventilation pipelines, most studies have focused on sparse discrete-phase particles. Limited research has been conducted on dense fine particle flows such as solid cleaning agent powders. Mutual impacts of dense particles have to be addressed urgently. Therefore, the migration and propagation process of solid cleaning agent particles in pipelines and the influencing factors were analysed through the dense discrete particle model (DDPM) by using the finite element numerical analysis method, thereby providing theoretical references for the selection of a reasonable purifying scheme.

The rest of this study is organized as follows. Section 3 introduces the construction of the engineering model, selection of a theoretical model for simulation, and setting of injection particle parameters and boundary conditions. Section 4 presents the analysis of the results. Section 5 summarizes the conclusions.

\section{METHODOLOGY}

\subsection{Engineering analysis and model construction}

In a large and complex 3D air supply pipeline, a certain amount of powder microparticulate solid substance is injected into the pipeline to clean gases. The solid powder migrates and diffuses along the pipelines under the effect of airflows, thereby realizing the goal of airflow purification. In practical operation, holes for material feeding were opened on the narrowing section of the pipelines and powder was injected through gravity and the negative static pressure of pipelines. However, the practical purifying effect is not satisfactory. Thus, analysing the migration and concentration distribution of powder particles in the pipelines can provide guidance in practical operation. This problem belongs to the scope of gas-solid flows in complicated ventilation network. In simulation, the primary factors to be considered are the following: (1) injection and trajectory tracking of dense microparticles, (2) flowing and distribution of microparticles in gas phase, (3) effects of gas turbulence on solid-phase particles, and (4) influences of injection mode of solid particles. The secondary major factors that have to be considered include (1) effects of gravity and (2) striking and connection of pipelines.

Based on the preceding analysis, this study aims to simulate the movement and distribution of dense particle flows based on the finite element analysis software Fluent by using multiphase flow of the Euler and DDPM models. According to the physical form of air supply pipelines, the abstract geometric model of pipelines can be obtained (Fig. 1). All of the sizes correspond to practical sizes. 


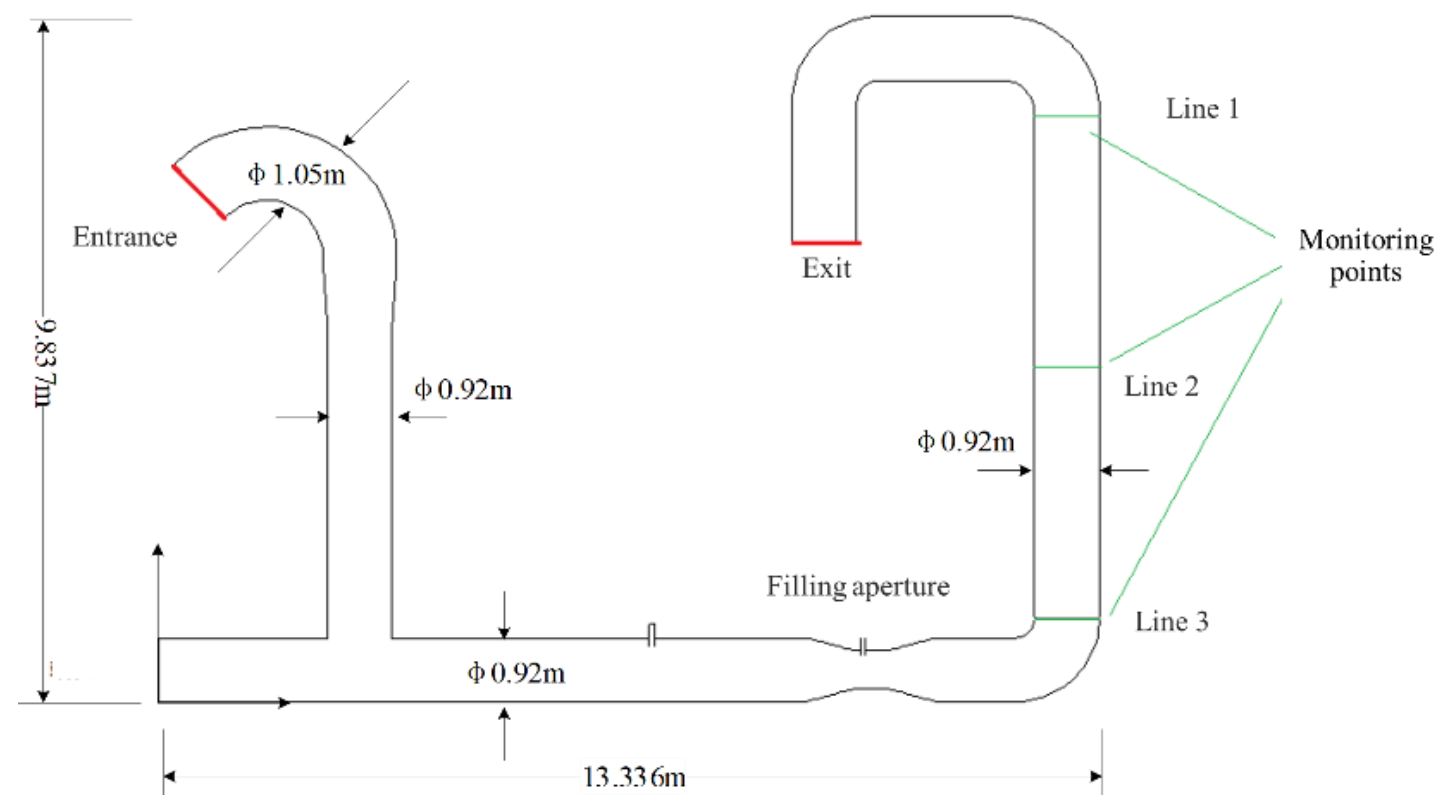

Figure 1: Geometric model.

Specifically, this model divides the smooth regions into several zones to refine the flow field characteristics of complex structural regions, simplifying the flow field characteristics of simple structural regions, and strengthening the division quality of networks. The refining degree among different blocks is determined by flow field structures. All blocks use the division method of structured grids. Results are shown in Fig. 2.

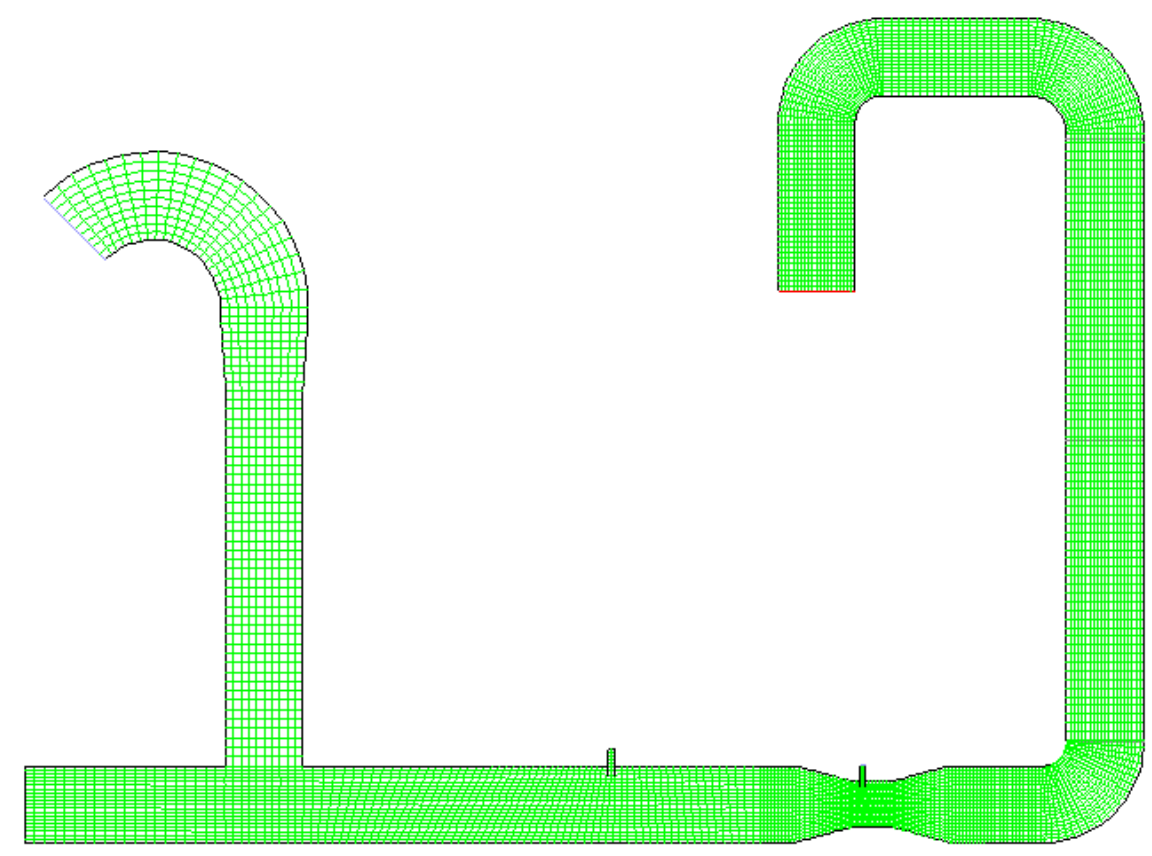

Figure 2: Grid mesh of model.

\subsection{Parameter setting and solving}

As the practical process involves the turbulence process of the gas phase and the collision and staking process of solid-phase thick particle flow, this study selected the $k-\varepsilon$ model to process turbulence process, standard wall function to process boundary flows, Euler model to simulate multiphase flow process, and DDPM model to process solid-phase particles. Parameter settings of dense particles added from the inlet are shown in Table I. 
Table I: Parameter settings for injected particles.

\begin{tabular}{|c|c|c|c|}
\hline Model parameter & \multicolumn{2}{|c|}{ Parameter values } & Unit \\
\hline \multirow{2}{*}{ Particle injection range } & $x$ & $9.952-10.02$ & \multirow{2}{*}{$\mathrm{m}$} \\
\hline & $y$ & $0.735-0.735$ & \\
\hline \multirow{2}{*}{ Initial particle velocity (assumed velocity) } & $x$ & 0 & \multirow{2}{*}{$\mathrm{m} / \mathrm{s}$} \\
\hline & $y$ & -10 & \\
\hline Injection particle density & \multicolumn{2}{|r|}{2400} & $\mathrm{~kg} / \mathrm{m}^{3}$ \\
\hline Particle mass flow & \multicolumn{2}{|r|}{0.4} & $\mathrm{~kg} / \mathrm{s}$ \\
\hline Maximum particle diameter & \multicolumn{2}{|r|}{30} & $\mu \mathrm{m}$ \\
\hline Minimum particle diameter & \multicolumn{2}{|r|}{20} & $\mu \mathrm{m}$ \\
\hline Mean particle diameter & \multicolumn{2}{|r|}{25} & $\mu \mathrm{m}$ \\
\hline Distribution index (Rosin-Rammler distribution) & \multicolumn{2}{|r|}{9.6} & -- \\
\hline Tracking the number of particle streams & \multicolumn{2}{|r|}{1000} & -- \\
\hline Method of particle injection & \multicolumn{2}{|r|}{ group } & -- \\
\hline
\end{tabular}

Boundary condition settings of the model mainly include those of air supply inlet, outlet, and injection mouth. Initial conditions of the model include gravity parameters, reference pressure, initial flow rate of flow field, pressure, and components. Major parameter settings are shown in Table II.

Table II: Main parameter setting of boundary and initial conditions.

\begin{tabular}{|l|c|c|}
\hline \multicolumn{1}{|c|}{ Model parameter } & Parameter type & Parameter values \\
\hline Air supply inlet (gas phase) & Speed entrance & $6.28,8.28,10.28 \mathrm{~m} / \mathrm{s}$ \\
\hline Return wind outlet (mixed phase) & Pressure exit & $0 \mathrm{~Pa}$ \\
\hline Injection port (gas phase) & Wall surface, speed inlet & $0,10 \mathrm{~m} / \mathrm{s}$ \\
\hline Acceleration of gravity & Y negative direction & $-9.81 \mathrm{~m} / \mathrm{s}^{2}$ \\
\hline Ambient pressure & Atmosphere & $101325 \mathrm{~Pa}$ \\
\hline
\end{tabular}

\section{RESULT ANALYSIS AND DISCUSSION}

\subsection{Flow field spatial distribution and particle migration law in narrowing zone under injection method}

Pressure and turbulence intensity distribution in the pipeline is shown in Fig. 3.
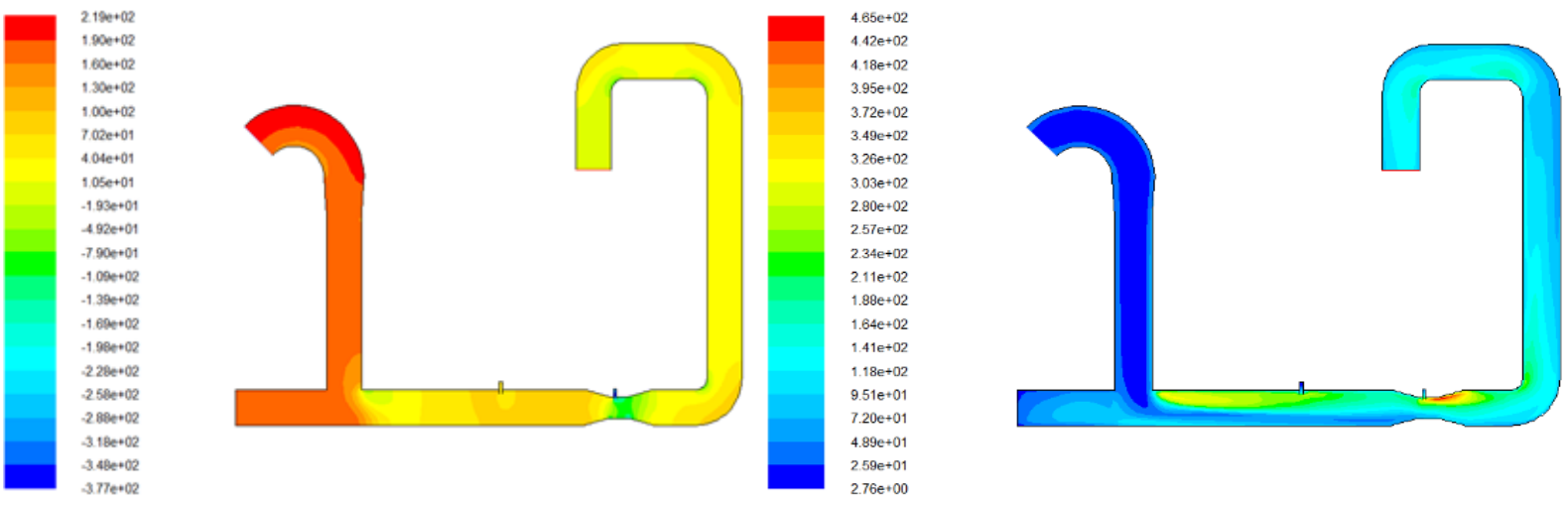

a) Static pressure

b) Turbulent intensity

Figure 3: Static pressure and turbulent intensity distribution of flow field after injection.

Fig. 3 a shows that when influenced by geometric structural changes of pipelines, pressure generally declines from the pipeline inlet to the outlet and an evident low-pressure zone exists in the rear part of the pipeline bending as well as the narrowing zone. Furthermore, the static pressure in the narrowing zone is negative, which leads to the theoretical feasibility of material 
injection method at the narrowing zone. Fig. $3 \mathrm{~b}$ shows an evident eddy region at the pipeline bending and downstream area of the material inlet. On the one hand, the existence of eddies can intensify the mixing degree between solid-phase particles and gas-phase flows. On the other hand, it causes an accumulation of materials in the region.

The gas-solid flow rate simulation is shown in Fig. 4. One can observe in Fig. 4 a that gas flow velocity in the pipeline is relatively stable and distributed uniformly in both pipeline trend and on the pipeline section except for an evident growth at the bending and narrowing zone. Fig. $4 \mathrm{~b}$ shows that since the inlet is at the top of the pipelines, the injected dense particles flow along the walls with airflow as a response to the viscosity force of the mainstream airflow in the pipeline. The flow velocity is closely related to the flow velocity of the gas-phase.

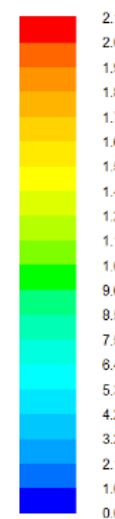

$2.15 \mathrm{e}+01$
$2.04 \mathrm{e}+01$
$1.93 \mathrm{e}+01$
$1.82 \mathrm{e}+01$
$1.72 \mathrm{e}+01$
$1.61 \mathrm{e}+01$
$1.50 \mathrm{e}+01$
$1.40 \mathrm{e}+01$
$1.29 \mathrm{e}+01$
$1.18 \mathrm{e}+01$
$1.07 \mathrm{e}+01$
$9.66 \mathrm{e}+00$
$8.59 \mathrm{e}+00$
$7.51 \mathrm{e}+00$
$6.44 \mathrm{e}+00$
$5.37 \mathrm{e}+00$
$4.29 \mathrm{e}+00$
$3.22 \mathrm{e}+00$
$2.15 \mathrm{e}+00$
$1.07 \mathrm{e}+00$
$0.00 \mathrm{e}+00$

a) Distribution of gas-phase velocity

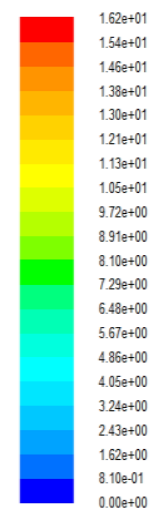

b) Distribution of solid-phase velocity

Figure 4: Flow velocity distribution of both gas-solid phases after injection.

The spatial distribution of particle concentration in the pipelines after injection stability is illustrated in Fig. 5.

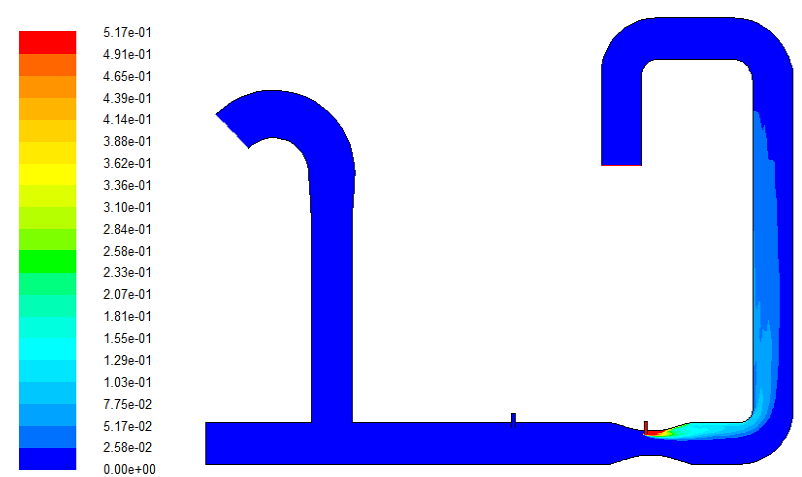

a) Distribution of particle concentration

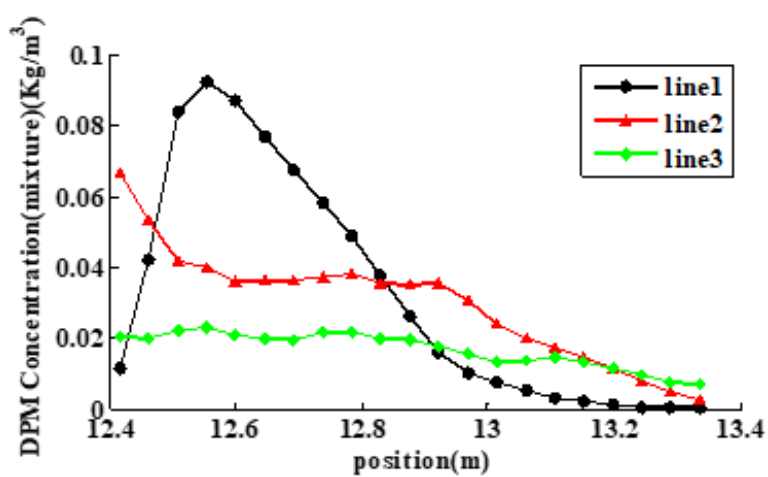

b) Particle concentration distribution curve

Figure 5: Concentration distribution of induced solid-phase particles after injection.

Fig. 5 a shows that except for the high particle concentration near the inlet, concentrations in other regions of the pipeline are relatively low and uneven distribution characteristics are obvious. The X-coordinate of the pipeline at the air return end ranges between 12.416 and 13.336. The distance of measuring line 1 from the bottom wall is $1.2 \mathrm{~m}$, that of line 2 is $4.8 \mathrm{~m}$, and that of line 3 is $8.4 \mathrm{~m}$. Both lines 1 and 3 are at the pipeline bending zone and line 2 is in the middle of the air return section. Fig. $5 \mathrm{~b}$ shows that after material injection, the material particle concentration presents typical proximal lateral distribution characteristics in the downstream sections of the major inlet. In other words, particle concentration on the pipeline wall near the inlet is significantly higher than those in other regions. According to the particle concentration distributions of measuring lines at different positions, the particle concentration in the pipelines generally declines with the increase in the transportation distance of the 
particles, and the typical proximal lateral distribution is improved gradually. This condition is mainly attributed to the influence of gas turbulence in the pipelines. However, the flow phenomenon along the wall remains. Obviously, the flow along walls caused by the injection method can lead to uneven particle concentration distribution in the pipelines. This is the main reason for the unsatisfactory purifying effect in the practical test.

\subsection{Flow field spatial distribution and particle migration laws at narrowing zone under jetting method}

According to the preceding analysis, particles are injected into pipelines and may flow along walls due to the influence of airflow, thereby weakening the injection effect of materials. For this reason, jetting is applied at a speed of $10 \mathrm{~m} / \mathrm{s}$. The gas-flow velocity of the jetting method is presented in Fig. 6.

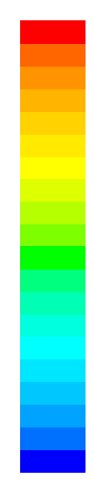

$2.81 e+01$
$2.67 e+01$
$2.53 e+01$
$2.39 e+01$
$2.25 e+01$
$2.11 e+01$
$1.97 e+01$
$1.83 e+01$
$1.69 e+01$
$1.55 e+01$
$1.41 e+01$
$1.27+01$
$1.13 e+01$
$9.84 e+00$
$8.44 e+00$
$7.03 e+00$
$5.63 e+00$
$4.22 e+00$
$2.81 e+00$
$1.41 e+00$
$0.00 e+00$

a) Distribution of gas phase velocity
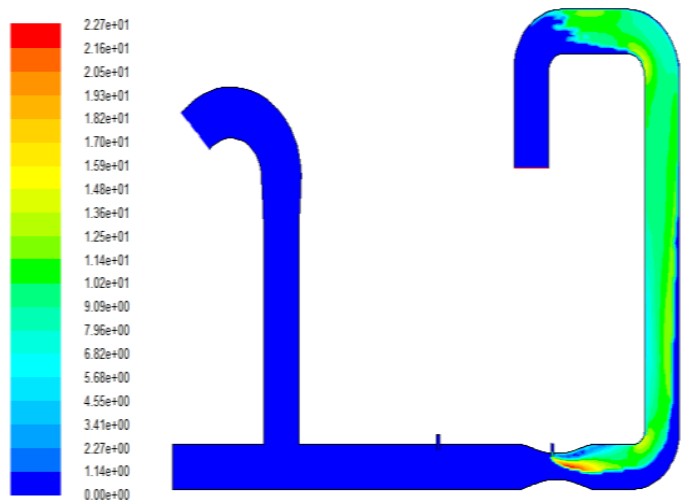

b) Distribution of solid phase velocity

Figure 6: Flow velocity distribution of both gas-solid phases after jetting method.

Fig. 6 a shows that the changing injection mode influences the flow velocity in the gas flow field slightly. According to a comparison of Figs. $6 \mathrm{~b}$ and $4 \mathrm{~b}$, the solid-phase particles at the inlet have obvious ejecting characteristics when the material feeding is changed from injection to jetting method, which improves the particle flow along the walls to a certain extent. However, Fig. $6 \mathrm{~b}$ shows that the flow velocity of material particles in the pipeline still occurs along walls.

After material jetting, the distribution cloud of the particle component concentration in the pipeline and the particle concentration distribution curve on various measuring lines in the air return section is shown in Fig. 7.

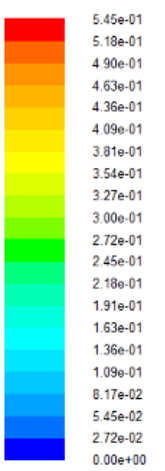

a) Distribution of particle concentration

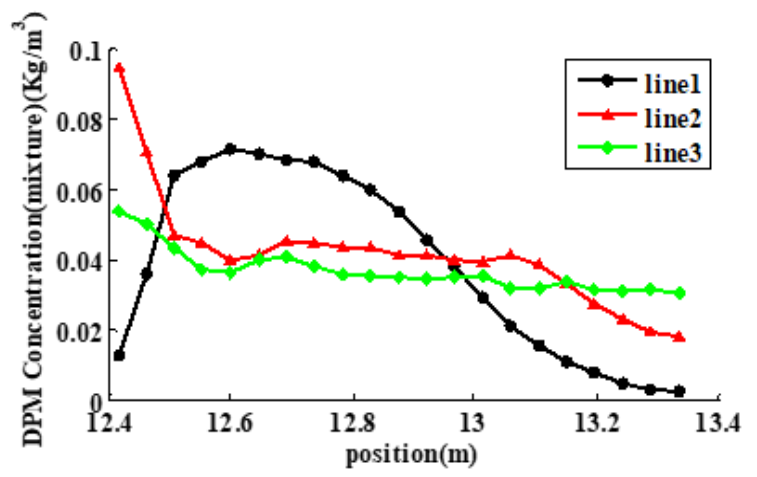

b) Particle concentration distribution curve

Figure 7: Concentration distribution of induced solid-phase particles after jetting method.

Fig. 7 a shows that under the jetting method of materials, the flow of particles along walls in the pipeline is improved to a certain extent and the particle concentration distribution in the middle of the pipeline at downstream areas generally occupies the entire section. Fig. $7 \mathrm{~b}$ shows 
that under the jetting method, the particle concentration distribution in the pipeline is still high on the near-wall side and low on the other side. However, the gap is decreased to a certain extent in view of numerical values where the mean of the concentration distribution in the pipeline is improved to some extent. Obviously, changing from the injection to the spraying method is beneficial for the mixing of particle and airflow and uniform distribution of particle concentration in pipelines.

\subsection{Effects of injection direction and position on material component concentration at narrowing zone}

Based on the preceding analysis, given the fixed flow field structure, changing the material injection into the jetting method can improve the purifying effect, but it is still not satisfactory. Given the fixed spraying flow rate, this study considered changing the spraying angle and area. The inlet is changed from the top to the middle of the pipeline, but the inlet diameter and spraying flow rate remain constant. Flow velocity distribution of the flow field is presented in Fig. 8.

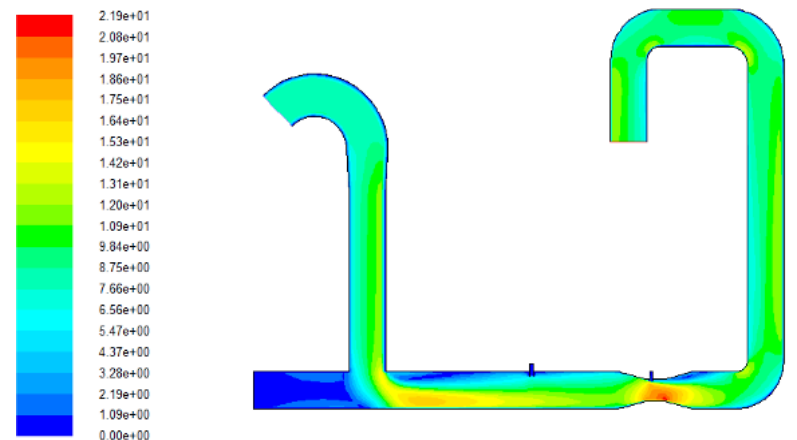

a) Distribution of gas phase velocity

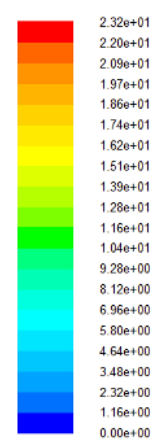

b) Distribution of solid phase velocity

Figure 8: Flow velocity distribution after changing injection direction and position.

Fig. 8 a shows that due to the high flow rate in pipelines, spraying materials hardly have influence on the structure of the gas-phase flow field, and any influence is negligible. In Fig. 8 b, one can observe that although some beamed flowing characteristics exist in the inlet area, particles spread over the entire section under turbulence after a very short distance of jet flows. The reason is that the spraying direction is consistent with the mainstream fluid and the inlet is at the turbulence area in the middle of the pipelines.

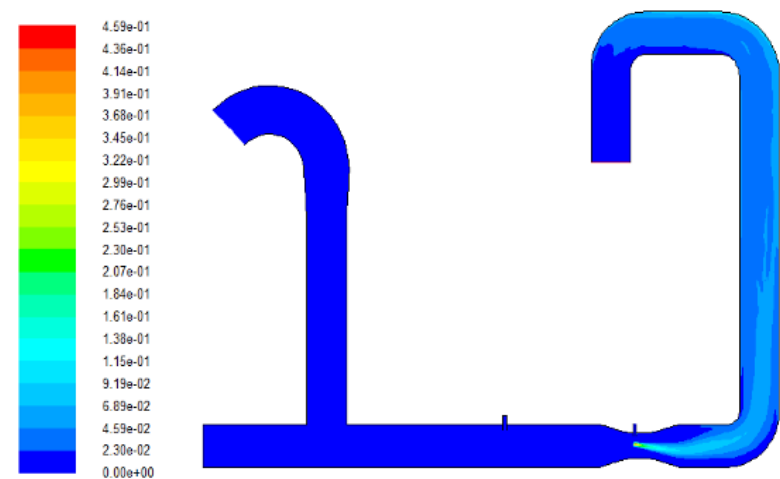

a) Distribution of particle concentration

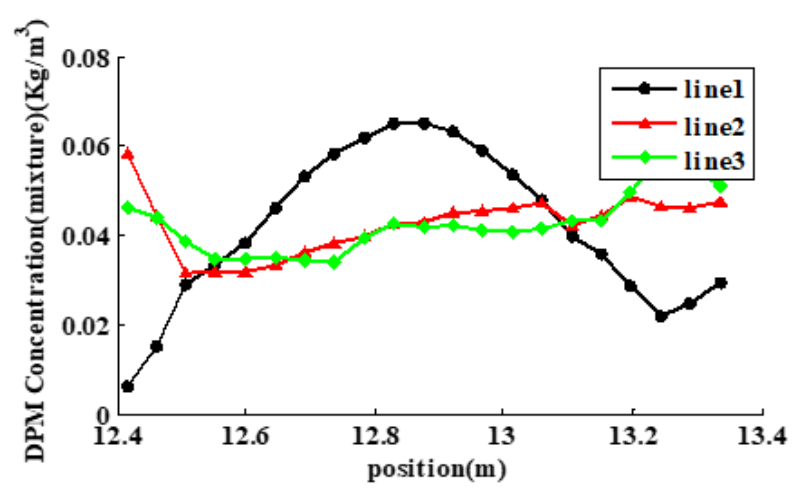

b) Particle concentration distribution curve

Figure 9: Concentration distribution of induced solid-phase particles after changing injection direction and position. 
Under the jetting method, particle component concentration distribution cloud in the pipeline and particle concentration distribution curves on different lines of the air return section are shown in Fig. 9.

Fig. 9 a shows that with changes in the injection direction and positions of materials, the material distribution increases gradually in the pipeline section. Furthermore, differences of component distribution between the pipeline bending and middle section are improved significantly, accompanied by some reduction of particle concentration in pipelines. However, the particle distribution in the pipelines still accounts for more than $50 \%$ of the pipeline areas, indicating that the concentrated flowing along walls is improved significantly. Fig. 9 b shows that the particle concentration at both inlet and air return section of the pipelines presents uniform distribution and the particle concentration in the pipelines is greatly increased. The flow characteristics along the walls are eliminated. Obviously, changing the inlet position and spraying direction can improve the flow characteristics along the walls and significantly weaken the uneven distribution of particle concentration in the pipelines.

\subsection{Effect comparison}

Based on the preceding analysis, uniform particle distribution in the pipelines and average particle concentration are two constraints against the purifying effect. In this study, measuring line 2 in the middle of the pipeline was used as the fundamental line. Mean concentration above measuring line 2 was used as the scale of concentration. The square of concentration values above measuring line 2 was used as the scale of the uniformity coefficient of concentration distribution. The injection method from the top of the narrowing section was used as the basic standard. The purifying effects of the aforementioned injection methods were compared. According to the characteristics of concentration and variance, the evaluation model adopted is as follows:

$$
\eta=\frac{c_{i} \times\left(-\ln \left(\operatorname{var}_{i}\right)\right)-c_{0} \times\left(-\ln \left(\operatorname{var}_{0}\right)\right)}{c_{0} \times\left(-\ln \left(\operatorname{var}_{0}\right)\right)} \times 100 \%
$$

In Eq. (1), $\eta$ is the overall improvement rate of scheme $i ; c_{i}$ is the mean concentration of the $i$ scheme; $v a r_{i}$ is the variance of the $i$ scheme concentration; and $c_{0}, v a r_{0}$ is the average concentration obtained by the injection method at the top of the tapered section and variance.

The evaluation results are presented in Table III.

Table III: Mean concentration and concentration variance under different injection schemes.

\begin{tabular}{|l|c|c|c|}
\hline \multicolumn{1}{|c|}{ Scheme } & $\begin{array}{c}\text { Mean concentration } \\
\left(\mathrm{kg} / \mathrm{m}^{3}\right)\end{array}$ & $\begin{array}{c}\text { Variance } \\
\left(\mathrm{kg} / \mathrm{m}^{3}\right)\end{array}$ & $\begin{array}{c}\boldsymbol{\eta} \\
(\%)\end{array}$ \\
\hline Injection method, top & 0.033204764 & $1.017699 \mathrm{E}-03$ & 0 \\
\hline Jetting method, top & 0.041701338 & $2.56079 \mathrm{E}-04$ & 0.507383873 \\
\hline Jetting method, central & 0.042272043 & $4.16555 \mathrm{E}-05$ & 0.863556647 \\
\hline
\end{tabular}

Table III shows that the average concentration and concentration square in the middle of the pipeline under the various injection methods are significantly different. The square under the injection method from the top of the narrowing section is the highest, indicating the highest nonuniformity of particle distribution. The average concentration and square under the jetting method in the middle of the narrowing section are the lowest, indicating that the jetting method is the optimal scheme theoretically. Table III also shows that the injection method at the mainstream region, both from the top and middle positions, brings a small average concentration and square. Thus, the method can effectively improve the distribution unevenness in theory. However, the average concentration is relatively small, which implies the great attenuation of material flows at the narrowing section. 
The calculation method that evaluates parameters based on different injection methods disclosed the effective improvement rates of the various injection methods compared with that of the jetting method at the narrowing section (Table III). Intuitively, the improvement rates of the other two schemes are positive compared with that of the injection method from the top of the narrowing section. The effective improvement rate of the jetting method from the middle of the narrowing section is the highest at $86 \%$, which conforms to the preceding analysis results.

\section{CONCLUSION}

To increase the purifying effect of pipelines by microparticles effectively and disclose the migration laws of dense particle flows in the pipelines with gas-solid migration, this study simulates the gas-solid flow velocity and particle concentration distribution in ventilation pipelines after the injection of microparticles by different ways and positions of the narrowing zone using multiphase flow in the Euler and DDPM models. This simulation study is based on theories related to hydromechanics and aims to solve practical engineering problems. Moreover, simulation results under several injection modes are compared and the optimal injection method for pipeline cleaning is determined. The following conclusions can be drawn:

(1) Influenced by the viscosity force of mainstream airflow, particles injected from the top of the narrowing section flow along walls as the air flows. Such a phenomenon can lead to uneven particle concentration distribution in pipelines and is the major cause of unsatisfactory purifying effect in practical tests.

(2) Compared with the injection method from the top of the narrowing section, the jetting method from the top presents obvious ejecting characteristics of solid particles at the inlet. The particles and airflow can be mixed effectively, which is beneficial for the uniform distribution of particle concentration in the pipelines. The particle flow along the walls is improved to a certain extent.

(3) Compared with the jetting method from the top of the narrowing section, the jetting method from the middle eliminates the particle flow along walls and the particle concentration distribution is uniform in pipelines. This condition weakens the uneven particle concentration distribution in pipelines and significantly improves the purifying effect of the pipelines.

(4) Given the same cleaning agent, the effective purifying effect of the jetting method from the middle of the narrowing section is improved by $86 \%$ compared with that of the injection method from the top.

From the perspective of practical engineering problems, a numerical simulation study on microparticle migration laws under various injection methods and injection positions from the narrowing section of pipelines is conducted by constructing a model of physical ventilation pipelines. Results have important significance in disclosing the causes of poor purifying effect of the ventilation pipelines and designing pipeline purification schemes reasonably. In this study, 2D simulation rather than a 3D spatial simulation on the migration laws of solid particles in ventilation pipelines is conducted using Fluent software. Simulation results may differ from actual situations. In future studies, a 3D model of the pipelines will be constructed to explore the particle migration laws in $3 \mathrm{D}$ spaces.

\section{REFERENCES}

[1] Abando, J. C.; Elguezabal, I. Z. (2021). Intellectual property in waste management: a reference to address a future challenge, Dyna, Vol. 96, No. 4, 388-394, doi:10.6036/10105

[2] Fu, J. J. (2020). Interpretation of technical efficiency of industrial waste gas treatment and its influencing factors, Resource Conservation and Environmental Protection, Vol. 2020, No. 5, 83, doi:10.16317/j.cnki.12-1377/x.2020.05.072 
[3] Rashid, T. U. (2021). Ionic liquids: Innovative fluids for sustainable gas separation from industrial waste stream, Journal of Molecular Liquids, Vol. 321, Paper 114916, 26 pages, doi:10.1016/ j.molliq.2020.114916

[4] Wang, L.; Zhao, Y. (2008). Kinetics of sulfite oxidation in wet desulfurization with catalyst of organic acid, Chemical Engineering Journal, Vol. 136, No. 2-3, 221-226, doi:10.1016/ j.cej.2007.04.004

[5] Fazaeli, R.; Fard, N. E. (2020). Desulfurization of gasoline fuel via photocatalytic oxidation/adsorption using $\mathrm{NaX}$ zeolite-based under mild conditions: process optimization by central composite design, Russian Journal of Applied Chemistry, Vol. 93, No. 7, 973-982, doi: $10.1134 /$ S1070427220070058

[6] Good, K. D.; VanBriesen, J. M. (2020). Correction to coal-fired power plant wet flue gas desulfurization bromide discharges to U.S. watersheds and their contributions to drinking water sources, Environmental Science \& Technology, Vol. 54, No. 1, 624-625, doi:10.1021/ acs.est.9b07028

[7] Zhong, Y.; Gao, X.; Huo, W.; Luo, Z.-Y.; Ni, M.-J.; Cen, K.-F. (2008). A model for performance optimization of wet flue gas desulfurization systems of power plants, Fuel Processing Technology, Vol. 89, No. 11, 1025-1032, doi:10.1016/j.fuproc.2008.04.004

[8] Shen, S.; He, J.; Pan, M.; Zhou, Z.; Chao, F.; Liang, G. (2012). Effective removal of sulfur from high-sulfur coal prior to use by dry chlorination at low temperature, Journal of Hazardous Materials, Vol. 217-218, 116-122, doi:10.1016/j.jhazmat.2012.03.001

[9] Scala, F.; D’Ascenzo, M.; Lancia, A. (2004). Modeling flue gas desulfurization by spray-dry absorption, Separation and Purification Technology, Vol. 34, No. 1-3, 143-153, doi:10.1016/ $\underline{\mathrm{S} 1383-5866(03) 00188-6}$

[10] Yamamoto, M.; Komaki, S.; Nakajima, D.; Matsushima, N.; Liu, D.; Nishioka, M.; Sadakata, M. (2006). Enhancement of $\mathrm{Ca}(\mathrm{OH})_{2} / \mathrm{fly}$ ash sorbent for the dry-desulfurization process, Energy \& Fuels, Vol. 20, No. 5, 1901-1905, doi:10.1021/ef050438q

[11] Burgess, J. E.; Parsons, S. A.; Stuetz, R. M. (2001). Developments in odour control and waste gas treatment biotechnology: a review, Biotechnology Advances, Vol. 19, No. 1, 35-63, doi:10.1016/S0734-9750(00)00058-6

[12] Baibikov, F. A.; Vlasov, G. A.; Kozen, A. L.; Buravtseva, G. I.; Balduev, M. E. (2009). Deep catalytic treatment of waste gas from sulfur dioxide, nitrogen oxides and carbon monoxide at processing products of detoxication of toxic substances, Russian Journal of Applied Chemistry, Vol. 82, No. 9, 1721-1728, doi:10.1134/s1070427209090365

[13] Straka, M.; Khouri, S.; Rosova, A.; Caganova, D.; Culkova, K. (2018). Utilization of computer simulation for waste separation design as a logistics system, International Journal of Simulation Modelling, Vol. 17, No. 4, 583-596, doi:10.2507/IJSIMM17(4)444

[14] Liu, X.; Liu, J.; Chen, J.; Zhong, F.; Ma, C. (2021). Study on treatment of printing and dyeing waste gas in the atmosphere with Ce-Mn/GF catalyst, Arabian Journal of Geosciences, Vol. 14, No. 8, Paper 737, 6 pages, doi:10.1007/s12517-021-07074-7

[15] Park, J.-H.; Ahn, J.-W.; Kim, K.-H.; Son, Y.-S. (2019). Historic and futuristic review of electron beam technology for the treatment of $\mathrm{SO}_{2}$ and $\mathrm{NOx}$ in flue gas, Chemical Engineering Journal, Vol. 355, 351-366, doi:10.1016/j.cej.2018.08.103

[16] Yin, M.; Xu, L. J.; Dai, Y.; Yang, D.; Zhu, X. (2021). Flow characteristics of oil-guiding splash lubrication: simulation and experiment studies, International Journal of Simulation Modelling, Vol. 20, No. 2, 363-374, doi:10.2507/IJSIMM20-2-CO6

[17] Ahmadi, G.; Goldschmidt, V. W. (1971). Motion of particles in a turbulent fluid-the basset history term, Journal of Applied Mechanics, Vol. 38, No. 2, 561-563, doi:10.1115/1.3408827

[18] Hutchinson, P.; Hewitt, G. F.; Dukler, A. E. (1971). Deposition of liquid or solid dispersions from turbulent gas streams: a stochastic model, Chemical Engineering Science, Vol. 26, No. 3, 419-439, doi:10.1016/0009-2509(71)83016-6

[19] Tian, L.; Ahmadi, G. (2007). Particle deposition in turbulent duct flows-comparisons of different model predictions, Journal of Aerosol Science, Vol. 38, No. 4, 377-397, doi:10.1016/ j.jaerosci.2006.12.003 
[20] Tsuji, Y.; Oshima, T.; Morikawa, Y. (1985). Numerical simulation of pneumatic conveying in a horizontal pipe, KONA Powder and Particle Journal, Vol. 1985, No. 3, 38-51, doi:10.14356/ kona.1985009

[21] Wu, F.; Gao, W.; Zhang, J.; Ma, X.; Zhou, W. (2018). Numerical analysis of gas-solid flow in a novel spouted bed structure under the longitudinal vortex effects, Chemical Engineering Journal, Vol. 334, 2105-2114, doi:10.1016/j.cej.2017.11.166

[22] Zhou, G.; Wang, D. M.; Cheng, W. M.; Cao, S. (2013). Numerical simulation research on gas-dust flow field of forced-exhausted hybrid ventilation in whole rock mechanized heading face, Applied Mechanics and Materials, Vol. 336, 873-879, doi:10.4028/www.scientific.net/AMM.336-338.873

[23] Li, J.; Kuipers, J. A. M. (2002). Effect of pressure on gas-solid flow behavior in dense gas-fluidized beds: a discrete particle simulation study, Powder Technology, Vol. 127, No. 2, 173-184, doi:10.1016/S0032-5910(02)00116-X

[24] Zhou, H.; Mo, G.; Zhao, J.; Cen, K. (2011). DEM-CFD simulation of the particle dispersion in a gas-solid two-phase flow for a fuel-rich/lean burner, Fuel, Vol. 90, No. 4, 1584-1590, doi:10.1016/j.fuel.2010.10.017

[25] Liu, H. T. (2010). Numerical simulation of micro particle deposition and diffusion characteristics in gas-solid two-phase flow, PhD Thesis, Chongqing University, Chongqing

[26] Zhang, H. H. (2016). Numerical Study of Cooking Particle Distribution and Movement, Master Thesis, Harbin Institute of Technology, Harbin 